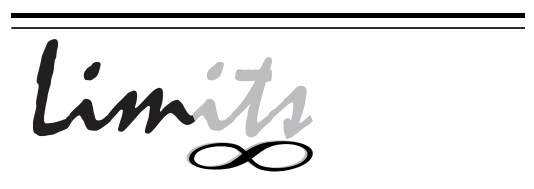

J. Math. and Its Appl.

ISSN : $1829-605 \mathrm{X}$

Vol. 7, No. 2, November 2010, 41-46

\title{
CRITICAL SET OF EDGE MAGIC TOTAL LABELING OF EXPANDING CYCLE GRAPH*
}

\author{
Chairul Imron ${ }^{1}$ and Suhud Wahyudi ${ }^{2}$ \\ Mathematics Department, FMIPA ITS Surabaya \\ ${ }^{1}$ imron-its@matematika.its.ac.id, ${ }^{2}$ suhud@matematika.its.ac.id
}

\begin{abstract}
This paper discusses about graph labeling. We will find edge magic total labeling of cycle and expanding cycle graph. In the final, we investigate the critical set of edge magic total labeling on cycle and expanding cycle graph.
\end{abstract}

Keyword: Cycle graph, edge-magic total labeling, critical set

\section{Introduction}

Let $G=(V, E)$ be a finite simple and undirected graphs. That is to say, they contain neither loops nor multiple edges. The graph have vertexset $V(G)$ and edge-set $E(G)$, and we denote $|V(G)|$ and $|E(G)|$ by $p$ and $q$ respectively. Graph Labeling is giving a label at vertices and edges of graph, so that each vertices and each edges having different label. Generally, label uses positive integer.

${ }^{*}$ This paper has been published on Names Conference 2009, UnLam, Banjarmasin, July $3^{\text {th }}-4^{\text {st }} 2009$. 
Several ways to labeling graph, labeling by domain from set of vertices, set of edges or both which generally referred as vertices labeling, edges labeling and total labeling. At this paper, we will present total labeling, especially edge-magic total labeling (EMTL) on cycle graph.

The edge-magic total labeling is sum of edges label and vertices label which adjacent at edges always equal for each edges. The sum is named magic number with symbol a letter $k$. Idea of labeling is defined first by [3] at 1960 and here in after [2] made a formula at 1970. A graph is called disconnected if its vertex-set can be partitioned into two subsets, $V_{1}$ and $V_{2}$, which have no common element, in such a way that there is no edge with one endpoint in $V_{1}$ and the other in $V_{2}$; if a graph is not disconnected, then it is connected. A cycle graph $C_{n}$ is connected graph that have $n$ vertex and $n$ edges.

Definition of edge-magic total labeling and super edge-magic total labeling:

Definition 1.1 [4] Edge-Magic Total Labeling An edge-magic total labeling of a $(p, q)$-graph $G$ is bijective function

$$
\lambda: V(G) \cup E(G) \rightarrow\{1,2,3, \ldots, p+q\}
$$

such that

$$
\lambda(u)+\lambda(u v)+\lambda(v)=k
$$

$k$ is a constant for any edge uv of $G$ and is called the magic sum of $G$. Any graph with an edge-magic total labeling will be called edge-magic. Morever, $\lambda$ is a super-edge-magic total labeling of $G$ if $\lambda(V(G))=\{1,2,3, \ldots, p+q\}$, and $G$ is said to be super-edge-magic.

For each graph, we number all vertex and edges, we call these numbers positions. Thus, a graph labeling can be represented as a set of ordered pairs of position and its label.

A critical set of a graph $G$ with labeling $\lambda$ is a set $Q_{\lambda}=\{(x, y) \mid x, y \in$ $\{1,2,3, \ldots, p+q\}$, with the oredered pair $(x, y)$ represents label $y$ in position $x$, which satisfy [5]

a. $\lambda$ is the only labeling of $G$ which has label $y$ in position $x$

b. No proper subset of $Q_{\lambda}$ 


\section{Edge-Magic Total Labeling}

Cycle graph is including graph regular, that is graph having same degree, usually notation of cycle graph with $C_{n}$. A cycle graph is connected graph that have $n$ vertices and $n$ edges.

Theorem 2.1 [1] Every cycle graph $C_{n}$ has an edge-magic total labeling with range of magic sum is

$$
\frac{5 n+3}{2} \leq k \leq \frac{7 n+3}{2}, \text { with } n \text { is odd }
$$

and

$$
\frac{5 n+4}{2} \leq k \leq \frac{7 n+2}{2} \text {, with } n \text { is even }
$$

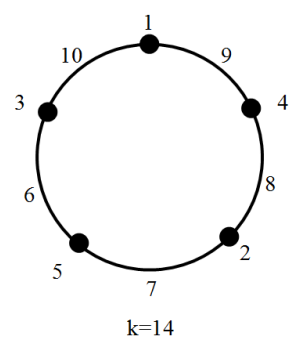

(a)

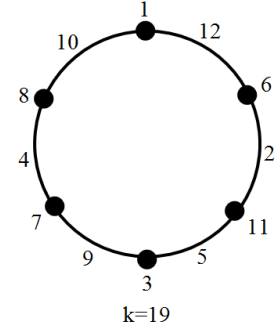

(b)

Figure 1: EMTL Cycle graph with $k=14$ and $k=19$

Theorem 2.2 Expanding cycle graph $C_{n}$ plus a edge has an edge-magic total labeling with range of magic sum is

$$
\frac{5 n^{2}+7 n+8}{2(n+1)} \leq k \leq \frac{7 n^{2}+17 n+4}{2(n+1)}
$$

Proof: Lets $S_{v}$ denote the sum of vertex labels and $S_{e}$ denote the sum of the edge labels in a edge-magic labeling $\lambda$. In the expanding cycle graph, sum of vertex is $p=n$ and sum of edge is $q=p+1$. Therefore, $k$ as magic sum of $G$ is

$$
\begin{aligned}
(n+1) k & =2 S_{v}+S_{e}+v_{i}+v_{j} \\
& =S_{v}+1+2+\ldots+(2 n+1)+v_{i}+v_{j} \\
& =S_{v}+2 n^{2}+3 n+1+v_{i}+v_{j}
\end{aligned}
$$


Among the labels, write $S$ for the set $\left\{v_{i} \mid 1 \leq i \leq n\right\}$ of vertex labels, and $k$ for the sum of elements of $S$. Then $S$ can consist of the smallest labels, the largest labels, or somewhere in between, so

$1+2+3+\ldots+n+3 \leq S_{v}+v_{i}+v_{j} \leq(n+2)+(n+3)+\ldots+(2 n+1)+4 n+1$

or

$$
\frac{n^{2}+n+6}{2} \leq S_{v}+v_{i}+v_{j} \leq \frac{3 n^{2}+11 n+2}{2}
$$

or

$$
\frac{5 n^{2}+7 n+8}{2(n+1)} \leq k \leq \frac{7 n^{2}+17 n+4}{2(n+1)}
$$

Theorem 2.3 Expanding cycle graph $C_{n}$ plus a edge has an super edgemagic total labeling with range of magic sum is

$$
\frac{5 n^{2}+7 n+12}{2(n+1)} \leq k \leq \frac{5 n^{2}+11 n+4}{2(n+1)}
$$

Proof: Lets $S_{v}$ denote the sum of vertex labels and $S_{e}$ denote the sum of the edge labels in a edge-magic labeling $\lambda$. In the expanding cycle graph, sum of vertex is $p=n$ and sum of edge is $q=p+1$. Therefore, $k$ as magic sum of $G$ is

$$
\begin{aligned}
(n+1) k & =2 S_{v}+S_{e}+v_{i}+v_{j} \\
& =2(1+2+\cdots+n)+(n+1)+(n+2)+\ldots+(2 n+1)+v_{i}+v_{j} \\
& =5 n^{2}+7 n+6+v_{i}+v_{j}
\end{aligned}
$$

Among the labels, write $S$ for the set $\left\{v_{i} \mid 1 \leq i \leq n\right\}$ of vertex labels, and $k$ for the sum of elements of $S$. Then $S$ can consist of the smallest labels, the largest labels, or somewhere in between, so

$$
1+2 \leq v_{i}+v_{j} \leq n+(n-1)
$$

or

$$
3 \leq v_{i}+v_{j} \leq 2 n-1
$$

or

$$
\frac{5 n^{2}+7 n+12}{2(n+1)} \leq k \leq \frac{5 n^{2}+11 n+4}{2(n+1)}
$$




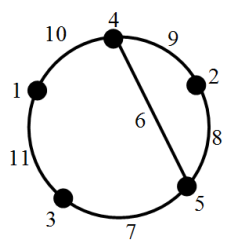

$\mathrm{k}=14$

(a)

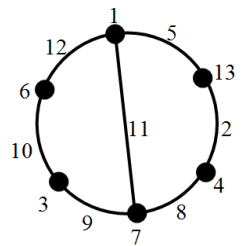

$\mathrm{k}=19$

(b)

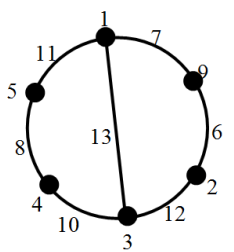

$\mathrm{k}=17$

(c)

Figure 2: EMTL Expanding Cycle Graph with $k=14, k=19$ and $k=17$

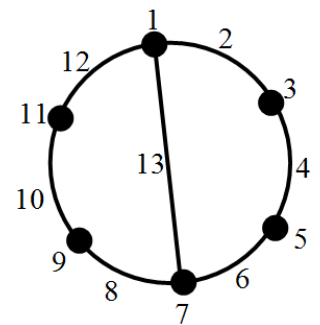

position

(a)

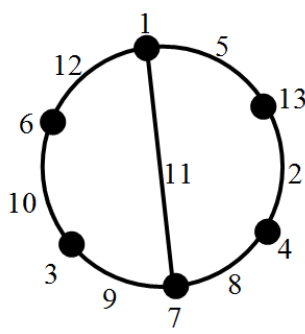

$\mathrm{L}_{1}$

(b)

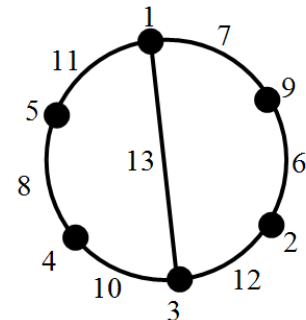

$\mathrm{L}_{2}$

(c)

Figure 3: Positon and Labeling of Expanding Cycle Graph

\section{Critical set}

Figure 3(a), shows of position label of vertices and edges, such that we have edgemagic total labeling of expanding cycle (Figure 3 (b)) with position is

$$
\begin{aligned}
L_{1}=\quad & \{(1,1),(2,5),(3,13),(4,2),(5,4),(6,8),(7,7), \\
& (8,9),(9,3),(10,10),(11,6),(12,12),(13,11)\}
\end{aligned}
$$

and position label of vertices and edges, such that we have edge-magic total labeling with position of another expanding cycle (Figure $3(\mathrm{~b})$ ) is

$$
\begin{aligned}
L_{1}=\quad & \{(1,1),(2,7),(3,9),(4,6),(5,2),(6,12),(7,3), \\
& (8,10),(9,4),(10,6),(11,5),(12,11),(13,13)\}
\end{aligned}
$$

If $Q$ is critical set and minimal number of critical set in expanding cycle graph that have many variation, big more and more expanding cycle more and more many 
critical set members and critical set of expanding cycle are

$Q_{1}=\{(1,1),(2,5)\}$

$Q_{2}=\{(1,1),(2,7)\}$

obviously after reconstruction, $Q_{2}$ have more than one edge-magic total labeling, which are

$$
\begin{aligned}
& L_{11}=\quad\{(1,1),(2,7),(3,9),(4,6),(5,2),(6,12),(7,3), \\
&(8,10),(9,4),(10,6),(11,5),(12,11),(13,13)\} \\
& \\
& L_{12}=\quad(1,1),(2,7),(3,12),(4,3),(5,5),(6,9),(7,6), \\
&(8,4),(9,10),(10,2),(11,8),(12,11),(13,13)\} \\
& \\
& L_{13}=\quad\{(1,1),(2,7),(3,12),(4,5),(5,3),(6,9),(7,8), \\
&(8,2),(9,10),(10,4),(11,6),(12,13),(13,11)\}
\end{aligned}
$$

so $Q_{2}$ is not critical set because the reconstruction is not unique, but $Q_{1}$ is critical set because reconstruction of $Q_{1}$ only one construction.

\section{Conclusion}

Critical set of expanding cycle graph depended on position and label of position, but expanding cycle graph in form of circle hence distance of position which and label influencing from to the number of members of critical set.

\section{References}

[1] Chairul Imron, Bandung A.S., Magic Graph on Cycle, The First International Conference on Mathematics and Statistics, UNISBA, Bandung, 19-21 Juni 2006.

[2] Kotzig, A. And A. Rosa, Magic Valuations of Finite Graph, Canad. Math. Bull. 13, 451-461, 1970.

[3] SedlaceK, J., problem 27, Theory of Graphs and it's Applications, 163-164, Publ. House Czechoslovak Acad. Sci.,Prague, 1964

[4] Wallis, W.D., E.T. Baskoro, M.Miller and Slamin, Edge-Magic Total Labelings, Australian Journal of Combinatorics 22, 177-190, 2000.

[5] E.T. Baskoro, Critical Sets in Edge-Magic Total Labelings, 2005. 